\title{
Chandra observations of the dwarf starburst and Wolf-Rayet galaxies NGC 4449 and NGC 5253
}

\author{
Lesley K. Summers, Ian R. Stevens \\ School of Physics and Astronomy, The University of Birmingham, \\ Edgbaston, Birmingham B15 2TT, UK
}

\author{
David K. Strickland, and Timothy M. Heckman \\ Department of Physics and Astronomy, The Johns Hopkins University, \\ 3400 North Carolina Street, Baltimore, MD 21218, USA
}

\begin{abstract}
We present an analysis of the Chandra observations of two dwarf starburst, Wolf-Rayet galaxies (NGC 4449 and NGC 5253). We have identified at least three different classes of objects within the X-ray point source populations, and we have found the diffuse emission, resulting from the stellar winds and supernova explosions of massive stars, to have a complex morphology and to consist of several components. Comparison with the $\mathrm{H} \alpha$ emission suggests the presence of $\sim \mathrm{kpc}$-scale wind-blown bubbles and ruptured superbubbles.
\end{abstract}

\section{Introduction}

X-ray observations of edge-on starburst (SB) galaxies (Strickland et al. 2000; Weaver 2001) present a picture of kpc-scale, bi-polar outflows, resulting from the activity of massive stars. Such pressure-driven galactic winds transport mass, newly synthesized heavy elements and energy into the galactic halo or on into the IGM. In dwarf SBs, such outflows can have a profound effect on the morphology and kinematics of the ISM and the galaxy's evolution. Chandra-ACIS-S3 observations of the two similar dwarf SB galaxies, NGC 4449 and NGC 5253, have identified a number of discrete point sources with spectra of different natures and at least two components within their diffuse emission.

\section{Results}

Within the $D_{25}$ ellipse, 24 sources were detected for NGC 4449 and 17 for NGC 5253 in the $0.3-8.0 \mathrm{keV}$ energy band. The $\log N$ vs. $\log L_{\mathrm{x}}$ plot of Figure 1 shows the range of their luminosities. Spectral analysis and hardness ratios for the NGC 4449 sources identify them with at least three different classes of objects, XRB, SNR and SSS. More high-luminosity sources are detected in NGC 4449 than in NGC 5253, and one difference between them is that NGC 5253 contains a very young SB at $\sim 3-4 \mathrm{Myr}$ (Schaerer et al. 1997). A single power law fit to the luminosity functions gives a slope of -0.38 for NGC 4449 , comparable to the value of -0.45 obtained for $\mathrm{M} 82$ and the Antennae galaxies (Zezas et al. 2001), while that for NGC 5253 is -0.74 . 

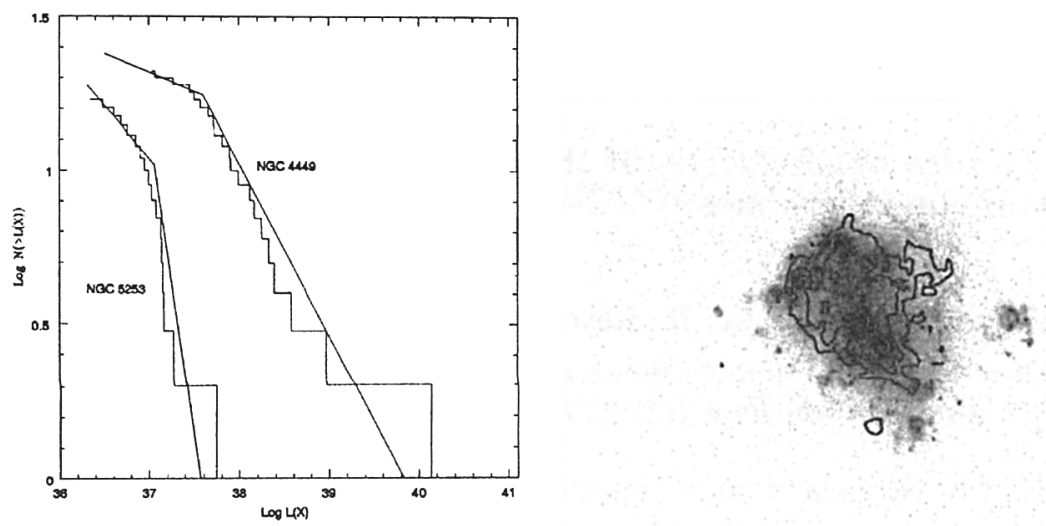

Figure 1. Left: $\log N$ vs. $\log L_{\mathbf{x}}$ plots of the resolved point sources within the $D_{25}$ ellipses of the two galaxies. Right: X-ray contours overlaid on an $\mathrm{H} \alpha$ image (kindly supplied by Deidre Hunter) of NGC 4449.

The diffuse emission is seen to be blobby in the central regions of both galaxies around star clusters and extends along their minor axes. Spectral fitting requires two thermal components with the softest one having $\mathrm{k} T \simeq 0.31 \mathrm{keV}$ for NGC 4449 and $k T \simeq 0.18 \mathrm{keV}$ for NGC 5253. The hotter components are both less extended and make a smaller contribution to the measured X-ray fluxes. For NGC $4449 \mathrm{k} T \simeq 0.82 \mathrm{keV}$, and for NGC 5253 it is hotter again with $\mathrm{k} T \simeq 3.1 \mathrm{keV}$. These two different gas components possibly reflect the expected presence of both shock-heated ISM material and shock-heated stellar wind/SN ejecta from the massive stars.

The morphologies and extent of both the $\mathrm{H} \alpha$ and diffuse X-ray emission are similar as shown in Figure 1. Surface brightness profile slices taken through both these emissions (Summers et al. 2002) suggests that the X-ray emission fills $\sim$ kpc-scale cavities in the $\mathrm{H} \alpha$ emission indicative of wind-blown superbubbles, and to the NW, the X-ray emission seems to extend beyond the $\mathrm{H} \alpha$ emission, possibly representative of the rupturing of a superbubble along the galaxy's minor axis. Analysis of XMM-Newton data for NGC 6263 is on-going and will hopefully help to clarify these results.

\section{References}

Schaerer, D., Contini, T., Kunth, D., Meynet, G. 1997, ApJ (Letters) 481, L75

Strickland, D.K., Heckman, T.M., Weaver, K.A., Dahlem, M. 2000, AJ 120, 1965

Summers, L.K., Stevens, I.R., Strickland, D.K., Heckman, T.M. 2002, in preparation

Weaver, K.A. 2001, in: J.H. Knapen, J.E. Beckman, I. Sholsman \& T.J. Mahoney (eds.), The Central Kpc of Starbursts and AGN: The La Palma Connection, ASP-CS 249, 389

Zezas, A., Fabbiano, G., Prestwich, A., Ward, M., Murray, S. 2001, in: J.H. Knapen, J.E. Beckman, I. Sholsman \& T.J. Mahoney (eds.), The Central Kpc of Starbursts and AGN: The La Palma Connection, ASP-CS 249, 425 